\title{
论文
}

\section{RC梁-柱中节点核心区剪切破坏及尺寸效应数值 分析}

\author{
金汶，隗娜，苗丽越，杜修力* \\ 北京工业大学城市减灾与防灾防护教育部重点实验室, 北京 100124 \\ *E-mail:duxiuli@bjut.edu.cn
}

收稿日期: 2018-12-27; 接受日期: 2019-04-30; 网络版发表日期: 2019-11-19

国家重点研发计划(编号: 2018YFC1504302, 2016YFC0701100)和国家自然科学基金(批准号: 51822801, 51421005)资助项目

\begin{abstract}
摘要钢筋混凝土梁-柱中节点核心区剪切破坏具有脆性特征, 因而其抗剪强度可能存在尺寸效应. 在已开展的 物理试验研究基础上，采用数值试验方法扩展讨论了结构尺寸(节点最大截面尺寸为 $900 \mathrm{~mm} \times 900 \mathrm{~mm}$ )、轴压比 和体积配䈐率对钢筋混凝土梁-柱中节点破坏机制与失效模式的影响, 并揭示了它们对剪切强度尺寸效应的影响 规律. 研究结果表明: (1) 单调加载下, 梁-柱中节点展现为核心区的脆性剪切破坏, 名义剪切强度具有明显尺寸效 应; (2) 轴压比的增大可提高中节点的抗剪承载力, 同时强化了剪切强度的尺寸效应; (3) 体积配䈐率的增大将增 强节点的抗剪承载力, 但会削弱剪切强度的尺寸效应. 此外, 经典的Bažant材料层次尺寸效应律可描述中节点剪 切破坏的尺寸效应行为, 但其无法描述轴压比与配䈐率的定量影响.
\end{abstract}

关键词梁-柱中节点, 核心区, 剪切破坏, 尺寸效应, 轴压比, 配䈨率, 数值模拟

\section{1 引言}

钢筋混凝土(reinforced concrete, RC)梁-柱节点作 为钢筋混凝土结构重要的传力机构, 受力复杂, 在地震 中极易发生脆性剪切破坏，造成难以修复的损伤甚至 导致坍塌, 是结构抗震的薄弱环节 ${ }^{[1,2]}$.

针对节点的受力性能及传力机理, 国内外研究者 开展了相关的试验研究, 如: 我国框架节点专题研究 组 ${ }^{[3]}$ 开展了 30 个试件的梁-柱节点核心区抗剪强度试 验研究; 傅剑平等人 ${ }^{[4]}$ 通过 10 组梁-柱节点试验探究了
受力机理及箍筋、轴压比等对节点的贡献及影响; 吕 西林等人 ${ }^{[5]}$ 研究了梁纵筋配筋率、核心区体积配䈨率 等的影响; Sadjadi和Kianoush ${ }^{[6]}$ 研究发现核心区配箍 量大的节点具有较好的抗剪性能; EI-Amour和Ghobar$\mathrm{ah}^{[7]}$ 进行了 12 个外节点试验, 探究了梁柱抗弯强度及 核心区箩筋的作用等. 这些研究工作表明, 影响节点 抗剪强度的因素很多, 除了混凝土材料本身的强度以 外, 还包括轴压比、配筢率、预应力、偏心影响及反 复荷载等因素. 其中, 轴压比及配䈨率是影响节点抗 剪承载力的关键因素.

引用格式: 金汶, 隗娜, 苗丽越, 等. RC梁-柱中节点核心区剪切破坏及尺寸效应数值分析. 中国科学: 技术科学, 2020, 50: 112-122 Jin L, Wei N, Miao L Y, et al. Numerical study on shear failure and the corresponding size effect of interior RC beam-column joints (in Chinese). Sci Sin Tech, 2020, 50: 112-122, doi: 10.1360/N092018-00441 
另外，钢筋混凝土梁-柱节点区的脆性破坏行为使 得其名义抗剪强度可能具有尺寸效应特征. 近些年来, 针对梁-柱节点破坏的尺寸效应行为, 也有研究者开展 了相关的试验工作, 如Abrams ${ }^{[8]}$, 李振宝等人 ${ }^{[9]}, \mathrm{Kim}$ 和 LaFave $^{[10]}$, Barbhuiya ${ }^{[11]}$ 和Choudhury等人 ${ }^{[12]}$ 的研究工 作, 这些试验结果证实了梁-柱节点的名义剪切强度具 有明显的尺寸效应行为. 总体来说, 这些少量的研究工 作促进了对梁-柱节点剪切破坏的认识. 尽管如此, 这 些工作依然是远远不够的，具体体现在如下方面：(1) 相关的试验工作，限于试验设备及经费，主要集中于 小尺寸构件破坏试验，缺乏对大尺寸(足尺)构件破坏 机制与失效模式的研究; (2) 缺少相关重要影响因素, 如轴压比、配箍率等对节点核心区剪切破坏尺寸效应 行为影响机制的认识; (3) 尚无梁-柱节点剪切破坏尺 寸效应律的相关模型与理论研究.

因此，针对梁-柱节点开展剪切破坏及其尺寸效应 行为试验与模拟分析，研究其破坏模式、失效机制及 节点强度的尺寸效应规律，探究其关键影响因素的作 用模式与机制，揭示其剪切破坏尺寸效应产生的物理 机制，进而为构建合理、科学的混凝土结构设计理论 与计算方法, 具有重要的科学和工程指导意义.

近来, Jin等人 ${ }^{[13]}$ 基于物理试验研究了单调和循环 加载下不同尺寸，尤其是大尺寸(最大节点截面尺寸

节点宽度 $\times$ 节点高度为 $900 \mathrm{~mm} \times 900 \mathrm{~mm}$ ) 钢筋混 凝土梁-柱中节点核心区剪切破坏试验，证实了节点核 心区剪切强度的尺寸效应行为, 并初步讨论了加载方 式等对剪切强度尺寸效应的影响规律与机制. 本文拟 在该梁-柱中节点剪切破坏试验基础上, 结合数值试验 方法更深入地讨论相关的重要因素，如轴压比及体积 配箙率等对节点核心区剪切破坏机制及剪切强度尺寸 效应规律的影响.

\section{2 已有试验工作概况}

近来，Jin等人 ${ }^{[13]}$ 开展了梁-柱中节点抗震性能及 尺寸效应研究的物理试验工作。该试验设计遵循构件 外形尺寸相似、抗剪承载力相似、正截面承载力相似 原则, 混凝土强度等级为 $\mathrm{C} 30$, 箍筋为 $\mathrm{HPB} 300$, 纵筋为 HRB400, 构件设计尺寸按 3:5:7:9比例分为9组(试件工 况和几何尺寸详见表 1 及表 2 , 表中的截面尺寸为最小 截面尺寸，长度为梁、柱的最长边，试件名称的命名 按照“BCJ-节点核心区宽度”), 采用不同轴压比、配䇽 率，探究了中节点在不同条件下的抗震性能及尺寸效 应，包括在梁端单调加载作用下，截面尺寸对节点的 抗剪承载力、核心区变形等性能的影响.

该试验结果 ${ }^{[13]}$ 表明: 节点名义剪切强度随结构尺 寸增大而减小, 尺寸效应显著, 随配䈐率提高, 节点的 抗剪承载力及变形能力增强. 具体的试验参数及相关 的研究结果, 可详见文献[13].

本文拟采用数值试验方法, 以该试验为基础, 扩展 模拟以研究更多参数对梁-柱中节点剪切破坏尺寸效 应的影响机制与规律. 鉴于此, 选取了 4 种节点尺寸(节 点核心区尺寸分别为 $300 \mathrm{~mm} \times 300 \mathrm{~mm}, 500 \mathrm{~mm} \times$ $500 \mathrm{~mm}, 700 \mathrm{~mm} \times 700 \mathrm{~mm}, 900 \mathrm{~mm} \times 900 \mathrm{~mm}$ )，5组不 同轴压比 $(0,0.15,0.3,0.45,0.6)$ 及3组不同配䈐率 $(0$, $0.42 \%, 0.84 \%$ ), 即共 60 组不同组合的梁-柱中节点剪切 破坏及尺寸效应的数值试验. 下文将讨论数值模拟方 法的选取与确定.

\section{3 梁-柱节点破坏的模拟方法}

\section{1 梁-柱节点宏/细观数值模型}

对于钢筋混凝土构件而言，其尺寸效应本质上是

表 1 试件工况

Table 1 Working conditions of specimens

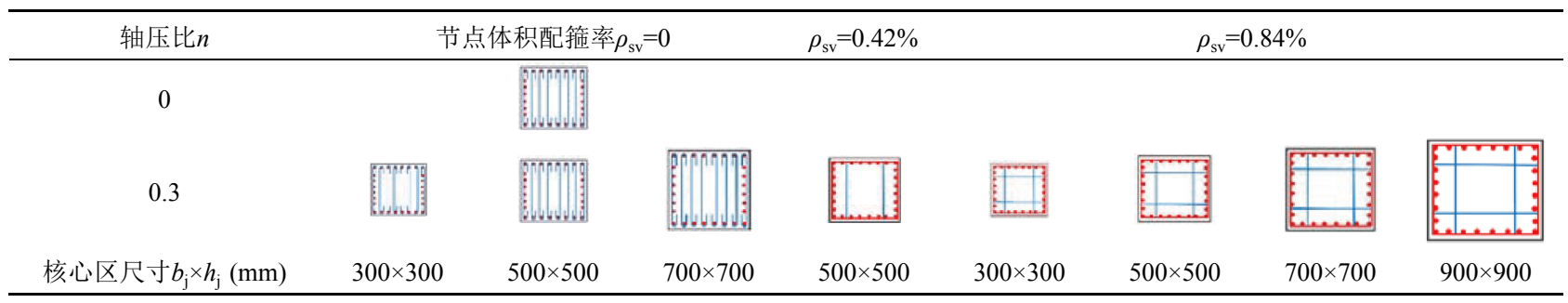


表 2 试件几何尺寸

Table 2 Geometric size of specimens

\begin{tabular}{cccc}
\hline 试件名称 & 位置 & 截面尺寸 $(\mathrm{mm})$ & 长度 $(\mathrm{mm})$ \\
\hline \multirow{3}{*}{ BCJ-300 } & 梁 & $225 \times 450$ & 1200 \\
& 柱 & $300 \times 300$ & 1500 \\
& 核心区 & $300 \times 300$ & \\
& 梁 & $375 \times 750$ & 1950 \\
BCJ-500 & 柱 & $500 \times 500$ & 2500 \\
& 核心区 & $500 \times 500$ & \\
& 梁 & $525 \times 1050$ & 2900 \\
BCJ-700 & 柱 & $700 \times 700$ & 3500 \\
& 核心区 & $700 \times 700$ & \\
& 梁 & $675 \times 1350$ & 3300 \\
BCJ-900 & 柱 & $900 \times 900$ & 4500 \\
& 核心区 & $900 \times 900$ & \\
\hline
\end{tabular}

由两个重要因素所造成的，包括混凝土材料本身的非 均质性及钢筋与混凝土间复杂的相互作用 ${ }^{[14]}$. 因此, 在数值模型的建立中，需同时反映这两个方面的“特 征”: 对于混凝土而言, 为反映材料的非均质性特征, 需要从细观或微观角度来进行描述; 另外, 还需考虑 钢筋与混凝土间非线性黏结-滑移关系.

考虑到计算量问题，这里采用细观尺度模型来描 述混凝土的非均质性. 细观尺度上，混凝土可看作是 由骨料颗粒、砂浆基质和界面过渡区组成的复合材 料. 此外, 还建立了宏观尺度模型(即将混凝土看作均 匀各向同性体)，用作与细观模拟结果的对比，进而考 查细观非均质性对梁-柱节点核心区剪切破坏尺寸效 应的定量影响. 鉴于此，建立了钢筋混凝土梁-柱节点 的代表性数值计算模型, 包括宏观及细观模型, 分别如 图1(a)和(b)所示.

数值模型建立的关键点在于混凝土本构关系的选 取. 塑性损伤模型基于塑性理论和连续介质损伤力学 理论建立, 可以体现通过微裂缝变化反映出的材料的 强化、软化以及永久变形和刚度的退化. 学者们对塑 性损伤模型做了很多研究，如Kim和 $\mathrm{Abu} A \mathrm{Al}-\mathrm{Rub}^{[15]}$ 提 出的塑性-断裂理论，Lubliner等人 ${ }^{[16]}$ 创立的“巴塞罗 那”模型等. Lee和Fenves ${ }^{[17]}$ 基于以上研究改进的塑性 损伤模型假定混凝土破坏方法为拉伸损伤和压碎破 坏，该模型能够很好地描述复杂受力状态下混凝土的 塑性不可逆变形行为及损伤破坏行为, 得到了广泛的
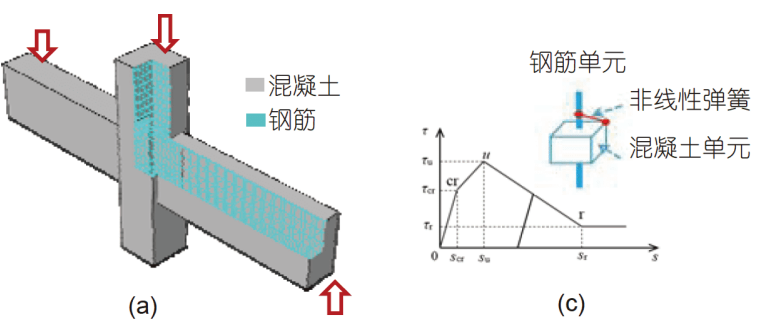

(c)
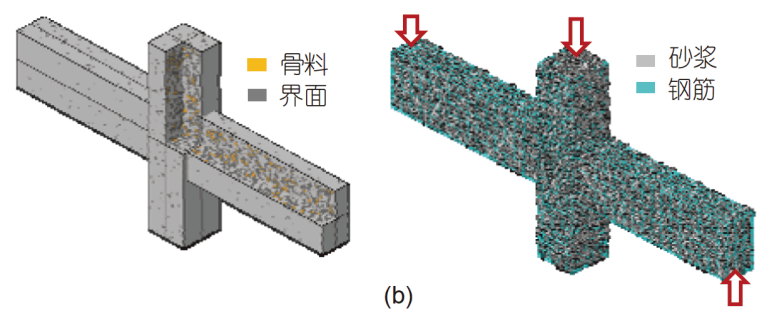

图 1 (网络版彩图)钢筋混凝土梁-柱节点三维宏细观数值 模型. (a) 宏观模型; (b) 细观模型; (c) 黏结-滑移处理

Figure 1 (Color online) Meso-scale numerical model of the RC beamcolumn joints. (a) Macro model; (b) meso-scale model; (c) bond stressslip relationship between concrete and steel rebar.

应用，如Genikomsou和Polak ${ }^{[18]}$ 关于混凝土板的冲剪 (punching shear)行为分析.

在本文建立的宏观尺度模型中，将构件视为由各 项均质的混凝土及钢筋两部分组成，混凝土采用塑性 损伤模型 ${ }^{[17]}$ 关系来描述其力学性能, 钢筋则采用理想 弹塑性模型. 细观尺度模型中, 采用Monte Carlo法随 机确定骨料的空间位置, 生成混凝土试件. 骨料所占体 积分数约为 $35 \%$, 骨料代表粒径为两种, 粗骨料大粒径 (30 mm直径)和细骨料小粒径(12 mm直径); 骨料周围 的薄层设为界面区, 其厚度为 $1 \mathrm{~mm}$. 参考文献[18], 砂 浆基质和界面过渡区采用能反映塑性永久变形及损伤 行为的塑性损伤模型 ${ }^{[17]}$ 来描述其力学行为; 骨料强度 高, 可认为其不产生破坏, 设为弹性体. 这里需要说明 的是, 为避免或减弱由于应变软化而带来的网格敏感 性问题，在该塑性损伤模型的使用中，材料拉伸应力应变关系曲线的下降段改用拉伸应力-位移关系曲线, 即通过采用断裂能唯一的方法来减弱网格敏感性问 题, 同 $\mathrm{Du}$ 等人 ${ }^{[19]}$ 研究工作.

上述宏-细观模型中, 混凝土采用八节点六面体实 体单元来划分网格, 平均网格尺寸为 $2.5 \mathrm{~mm}$; 钢筋采 用两节点线性三维桁架单元进行离散, 平均网格尺寸 为 $2.5 \mathrm{~mm}$.

关于钢筋与混凝土间的非线性相互作用问题，这 里钢筋与混凝土界面间的黏结-滑移行为采用我国 
《混凝土结构设计规范》 ${ }^{[20]}$ 推荐的方法, 即通过建立 非线性弹簧单元的方式来描述钢筋-混凝土间的非线 性黏结-滑移 $(\tau-s)$ 关系，如图1(c)所示. 该本构模型中, 各转折点参数的物理意义及相关取值方法, 可详见文 献[18].

\section{2 梁-柱节点破坏模拟方法验证}

为验证以上所述宏观及细观数值模拟方法的合理 性和准确性, 首先对文献[13]梁-柱节点试验进行对应 的宏-细观数值模拟.

选取的试验构件的相关参数: 节点核心区尺寸(节 点宽度 $\times$ 节点高度) 为 $300 \mathrm{~mm} \times 300 \mathrm{~mm}$ 、轴压比为 0.3 、配䇽率为 $0.84 \%$. 宏观及细观模型的外形尺寸及 配筋形式均与试验设计一致, 受力方式为柱端受恒定 轴向压力, 梁端受反向单调位移加载控制, 如图1(a)和 (b)所示.

需先确定混凝土细观组分(骨料、砂浆基质及界 面区)的力学参数, 包括弹性模量、拉伸/压缩强度、 泊松比及其他的相关参数等. 实际上, 由于界面过渡 区实际厚度很小 (约 $20 \sim 50 \mu \mathrm{m})$, 其力学参数很难通过 试验测试获得. 另一可替换的方法为反演法, 即通过 反复试算的方法来确定界面的力学参数. 本文首先建 立边长为 $150 \mathrm{~mm}$ 的非均质混凝土立方体试件, 对界面 区取砂浆力学参数的折减数值, 对混凝土试件的单轴 压缩及䢃拉破坏行为进行大量的数值试算, 发现采用 表 3 中给出的界面相及砂浆基质力学参数, 包括抗 压、抗拉强度及弹性模量等参数时, 模拟得到的混凝 土单轴抗压强度/䢃拉强度为 $34.2 \mathrm{MPa} / 3.10 \mathrm{MPa}$, 与试 验实测结果 $34.55 \mathrm{MPa} / 3.06 \mathrm{MPa}$ 基本吻合, 从而说明了 参数选取的合理性. 表 3 中, “太”所示的力学参数为实 测数据, “项”所示的界面力学参数(特别是材料弹性模

表 3 混凝土细观组分及钢筋力学参数 ${ }^{\text {a) }}$

Table 3 Mechanical parameters of concrete components and steel bars $^{\text {a) }}$

\begin{tabular}{|c|c|c|c|c|c|}
\hline 细观组分 & $\begin{array}{c}\text { 抗压强度 } \\
(\mathrm{MPa})\end{array}$ & $\begin{array}{c}\text { 抗拉强度 } \\
\text { (MPa) }\end{array}$ & $\begin{array}{c}\text { 弹性模量 } \\
\text { (GPa) }\end{array}$ & 泊松比 & $\begin{array}{c}\text { 屈服强度 } \\
(\mathrm{MPa})\end{array}$ \\
\hline 骨料 & - & - & $\star^{\star} 70$ & ${ }^{\star} 0.2$ & - \\
\hline 砂浆基质 & $\star^{\star} 36.17$ & $\star_{3.41}$ & $\star_{32.1}$ & ${ }^{\star} 0.2$ & - \\
\hline 界面过渡区 & 28.94 & 2.72 & 25.68 & 0.2 & - \\
\hline 钢筋 & - & - & $\star_{203}$ & ${ }^{\star} 0.3$ & $\star_{496}$ \\
\hline
\end{tabular}

a) ${ }^{\star}$ 为实测参数, 沉为反复试算参数.
量和强度)难以通过试验直接测得.

在上述力学参数下, 对选取节点的破坏行为进行 了细观数值模拟, 结果如图 2 所示. 图2(a)为宏观模型 损伤破坏图, 可以体现出节点受力后的剪切破坏特征, 即节点的破坏以核心区破坏为主，核心区出现斜向的 贯通裂缝, 其损伤在节点中心位置达到最大; 图2(b)为 细观模型损伤破坏图, 发生显著的核心区剪切破坏, 并 且破坏向梁端延伸, 与图2(c)中给出的试验破坏现象 吻合.

图3为该构件试验和模拟得到梁端荷载 $P$-位移 $\Delta$ 曲线对比图, 细观力学分析模型的模拟结果较宏观模 型结果与已有试验值吻合程度更高. 正如Du等人 ${ }^{[19]}$ 研 究表明, 宏观混凝土模型不能反映细观非均质性, 因而 不能够充分地反映钢筋混凝土构件的尺寸效应行为, 尤其对于钢筋混凝土构件破坏时混凝土部分起主导作 用情况, 如少(䈨)筋或超(笮)筋混凝土梁中发生剪切破 坏行为. 因此, 在接下来的数值研究中, 选用细观模拟 作为研究节点剪切破坏及尺寸效应的数值方法.

\section{4 模拟结果及分析}

\section{1 模拟结果}

在试验的基础上，对4种节点核心区尺寸 $(300 \mathrm{~mm}$ $\times 300 \mathrm{~mm}, \quad 500 \mathrm{~mm} \times 500 \mathrm{~mm}, \quad 700 \mathrm{~mm} \times 700 \mathrm{~mm}$,

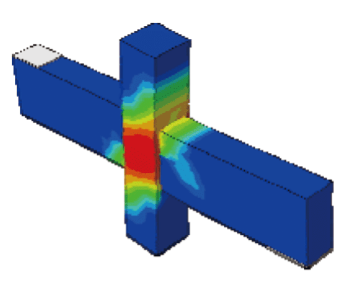

(a)

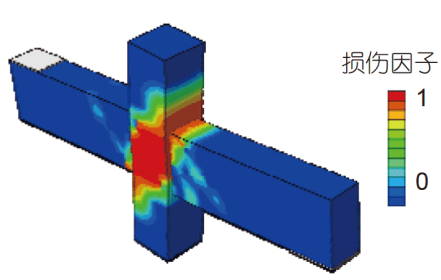

(b)

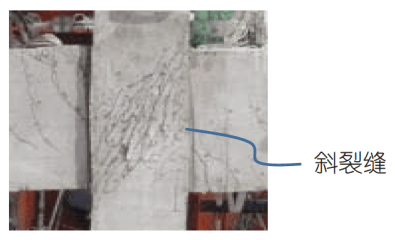

(c)

图 2 (网络版彩图)钢筋混凝土梁-柱节点(节点截面宽度 $300 \mathrm{~mm}$ )损伤破坏. (a) 宏观模型; (b) 细观模型; (c) 试验试件 损伤破坏

Figure 2 (Color online) Failure model of the RC beam-column joints (width of joint section is $300 \mathrm{~mm}$ ). (a) Macro model; (b) meso-scale model; (c) failure model of test. 


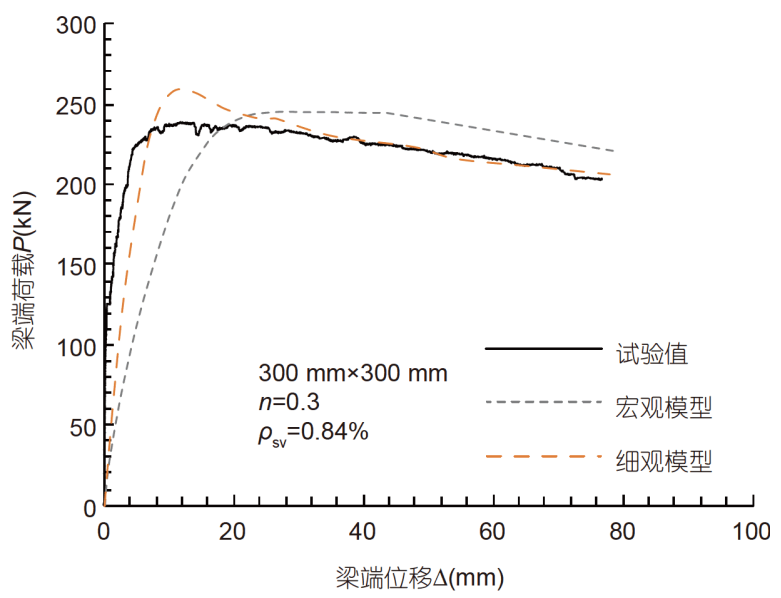

图 3 (网络版彩图)梁端荷载 $P$-位移 $\Delta$ 曲线对比

Figure 3 (Color online) Comparison of beam end load $P$-displacement $\Delta$ curves.

$900 \mathrm{~mm} \times 900 \mathrm{~mm}) 、 5$ 种轴压比 $(0,0.15,0.3,0.45,0.6)$ 及3种配箙率 $(0,0.42 \%, 0.84 \%)$ 工况下的中节点进行了 细观数值模拟. 图 4 为节点核心区宽度 $b_{\mathrm{j}}$ 分别为 300 , 500,700 和 $900 \mathrm{~mm}$ 的节点梁端荷载 $P$-位移 $\Delta$ 曲线模拟 值及试验值对比图，良好的吻合情况进一步验证了模 拟方式的合理性. 同时, 随着节点尺寸增大, 梁端荷载 在经历峰值后的下降段从较为平缓发展为迅速下降, 说明试件延性随尺寸增大而降低.

图5为轴压比 0.3 、配䈐率 $0.84 \%$ 情况下，不同尺寸 节点的损伤破坏图. 各尺寸的节点均表现出了明显的 核心区剪切破坏形式，但随尺寸的增大，节点的损伤 破坏范围扩大，呈现出由节点核心区逐渐向梁端及柱 端延伸的趋势. 出现这种情况的原因归结于节点在受

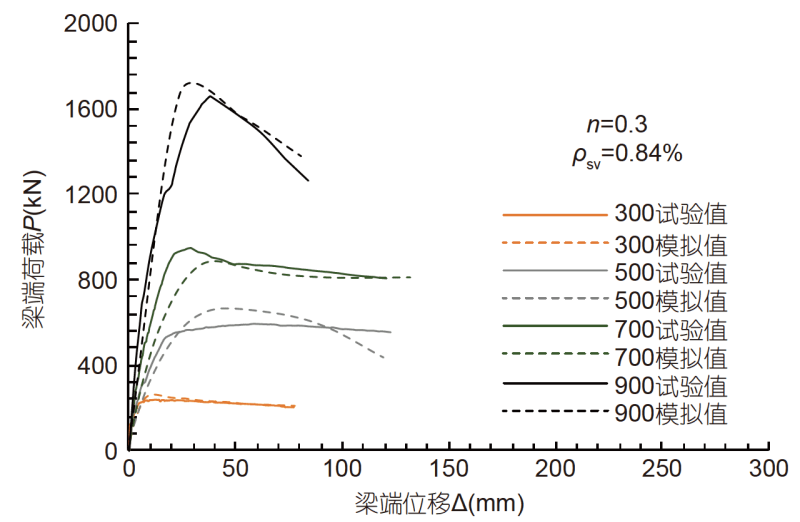

图 4 (网络版彩图)梁端荷载 $P$-位移 $\Delta$ 曲线对比

Figure 4 (Color online) Comparison of beam end load $P$-displacement $\Delta$ curves.

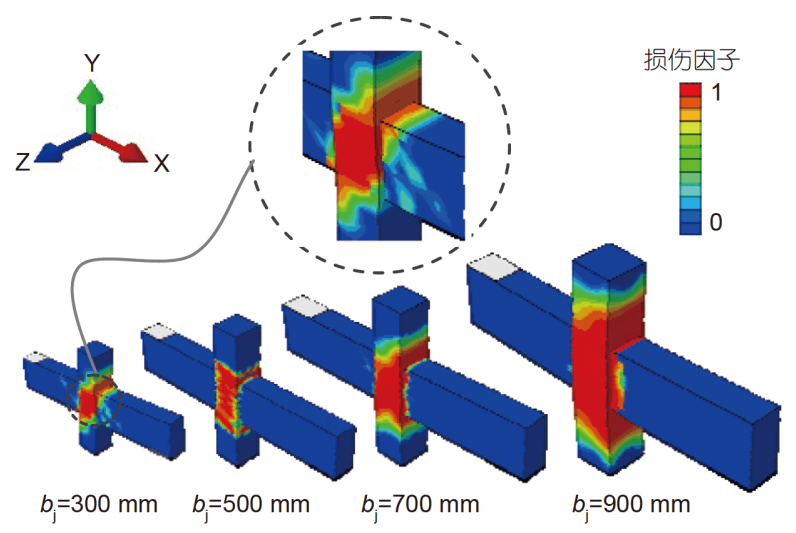

图 5 (网络版彩图)不同结构尺寸的梁-柱节点损伤破坏图 Figure 5 (Color online) Failure models of the RC beam-column joints with different structural sizes.

力后，核心区的强度和刚度退化，大尺寸构件受尺寸 效应影响，其强度弱于较小尺寸的构件，因此破坏范 围明显增大。

试验与模拟获取的是梁端的荷载 $P$-位移 $\Delta$ 曲线, 为探究中节点所受剪力的尺寸效应问题，根据《混凝 土结构设计规范》 ${ }^{[20]}$ 中关于 $\mathrm{RC}$ 梁-柱节点核心区的计 算公式计算核心区所受的实际剪力 $V_{\mathrm{jt}}$ :

$V_{\mathrm{jt}}=\sum \frac{M_{\mathrm{b}}}{h_{\mathrm{b} 0}-a_{\mathrm{s}}^{\prime}}\left(1-\frac{h_{\mathrm{b} 0}-a_{\mathrm{s}}^{\prime}}{H_{\mathrm{c}}-h_{\mathrm{b}}}\right)$,

式中， $M_{\mathrm{b}}$ 为节点左右两侧的梁端反时针或顺时针方向 组合弯矩设计值之和, $h_{\mathrm{b} 0}$ 为梁截面有效高度, $a_{\mathrm{s}}^{\prime}$ 为梁 纵向受压钢筋合力点至截面近边的距离, $H_{\mathrm{c}}$ 为节点上 柱和下柱反弯点之间的距离, $h_{\mathrm{b}}$ 为梁截面高度.

采用名义剪切强度来探讨节点在不同尺寸、不同 轴压比及配䈐率下的抗剪性能, 名义剪切强度 $\tau_{\mathrm{u}}$ :

$\tau_{\mathrm{u}}=V_{\mathrm{jt}} / A_{\mathrm{j}}$,

式中, $A_{\mathrm{j}}$ 为核心区面积.

经过计算得出了所有工况下中节点的名义剪切强 度, 如图6所示, 横坐标为节点核心区宽度 $b_{\mathrm{j}}$, 纵坐标为 名义剪切强度, 不同图例代表不同轴压比, 同种图例沿 纵坐标向上代表配䈨率由小变大. 可以看出, 随节点尺 寸增大, 中节点的名义剪切强度有明显的下降趋势, 即 中节点剪切破坏存在尺寸效应. 另外, 图6也同时反映 出轴压比及配䈐率随尺寸变化而体现出的名义剪切强 度变化规律, 如轴压比的增大会增强尺寸效应, 配箍率 的提高削弱尺寸效应等，下文将对此进行详细分析与 讨论. 


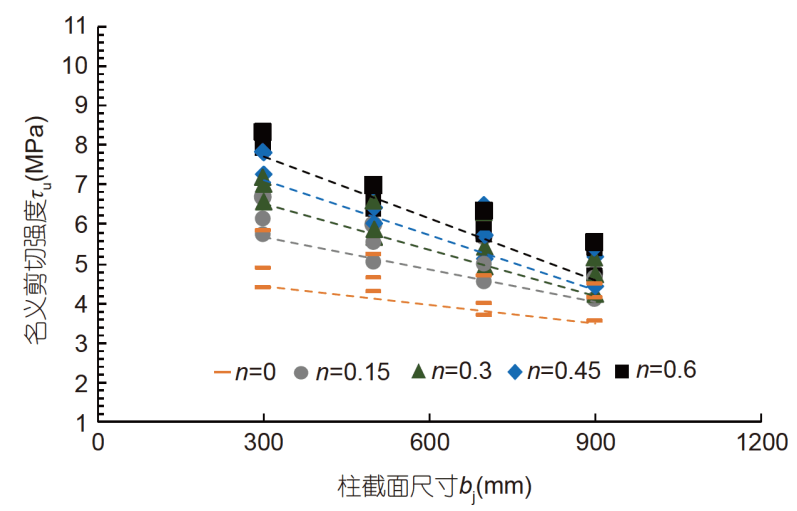

图 6 (网络版彩图)名义抗剪强度与节点宽度的关系

Figure 6 (Color online) The relationship between nominal shear strength and joint width.

\section{2 轴压比影响}

柱轴压比代表由柱端传入节点的轴压力大小，可 以影响主压应力大小及钢筋的黏结条件从而改变节点 核心区力学性能. 为了更直观地描述轴压比及配䈐率 对节点力学性能的影响, 将模拟所获取的梁端荷载 $P$ 位移 $\Delta$ 曲线中的横坐标一梁端位移 $\Delta$ 替换为梁端位移 角 $\theta$, 该位移角为梁端坚向位移与梁有效臂长的比值, 代表梁与柱的相对转角，能够体现不同尺寸节点的延 性及变形能力及其差异.

图7为配箙率为 $0.42 \%$, 不同尺寸、不同轴压比下 的节点梁端荷载 $P$-位移角 $\theta$ 曲线图. 各尺寸的节点梁端 峰值荷载均随轴压比的增大而增大; 较小尺寸的节点 峰值荷载后的下降段较平缓，即试件的延性较好; 大 尺寸节点在达到峰值荷载后即迅速下降，出现显著的 脆性破坏特征.

图8为节点宽度为 $300 \mathrm{~mm}$ 、配筷率 $0.84 \%$, 不同轴 压比情况下的节点损伤破坏图. 可以看出, 随轴压比增 大, 节点破坏区域有所扩大, 当轴压比较大时, 节点破 坏产生的斜裂缝向上下柱端内发展。在节点受到梁端 传递来的弯矩(剪力)作用时，节点核心区从中部产生 细微裂缝并逐渐发展为斜向大裂缝，最终因核心区混 凝土压碎而导致构件破坏失效。而在柱端施加的轴向 作用力可以在开裂初期增大各相介质之间的咬合力, 降低裂缝发展速度, 进而增强节点的抗剪能力, 但较大 的轴压比会降低节点的延性. 值得注意的是, 如果所承 受的轴压力更大(如轴压比达到 0.8 ), 则可能导致混凝 土被直接压碎, 对节点抗剪强度的提高是不利的.
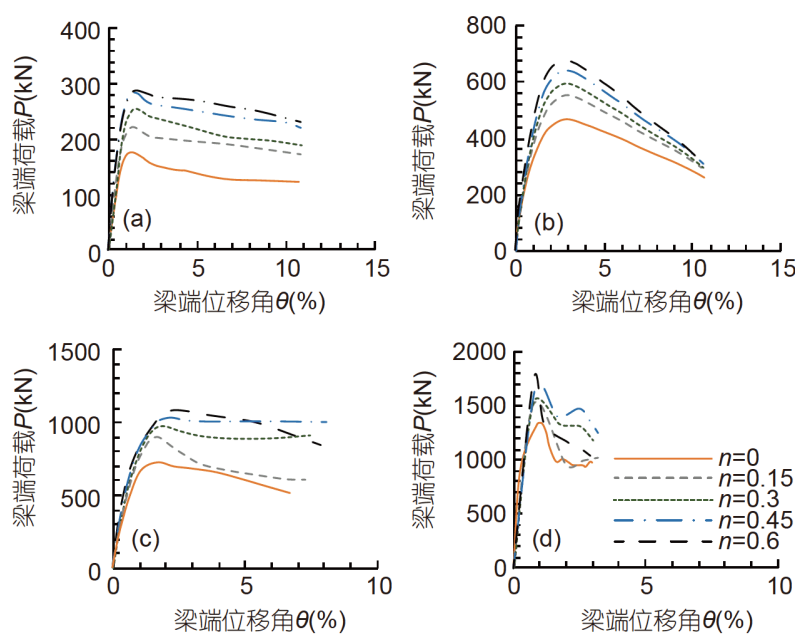

图 7 (网络版彩图)配䈐率为 $0.42 \%$ 时梁端荷载 $P$-位移角 $\theta$ 曲 线对比. (a) 节点宽度 $300 \mathrm{~mm}$; (b) 节点宽度 $500 \mathrm{~mm}$; (c) 节点 宽度700 mm; (d) 节点宽度900 mm

Figure 7 (Color online) Comparison of load $P$ - displacement angle $\theta$ curves of beam end at $0.42 \%$ stirrup ratio. (a) Joint width $300 \mathrm{~mm}$; (b) joint width $500 \mathrm{~mm}$; (c) joint width $700 \mathrm{~mm}$; (b) joint width $900 \mathrm{~mm}$.

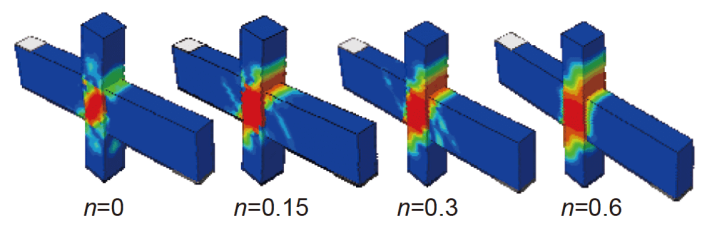

图 8 (网络版彩图)不同轴压比节点损伤破坏图

Figure 8 (Color online) Failure models of joints with different axial compression ratio.

为了说明轴压比对节点名义剪切强度的影响, 从 图6中选取配箍率为 $0.42 \%$ 的节点名义剪切强度数值, 从而展示不同轴压比下节点名义剪切强度随尺寸变化 的关系, 如图9所示. 可以看出, 同一轴压比下, 随尺寸 增大, 节点的剪切强度降低, 而随着施加在节点上轴压 比(0 0.6)的增大, 这种降低的趋势愈发显著, 即增大轴 压比增强了尺寸效应. 实际上, 轴压比越大, 节点破坏 时的脆性越强, 故而尺寸效应更为显著. 同时, 在同一 尺寸下, 随着轴压比的增大, 其节点的抗剪强度相应地 有所提高, 但这种提高程度随着尺寸的增大而削弱.

为更加直观地体现轴压比对节点抗剪强度的提高 程度, 本文定义名义剪切强度提高百分比 $Z, \tau_{\mathrm{u}}$ 代表某 一轴压比下的名义抗剪强度, $\tau_{\mathrm{u} 0}$ 代表轴压比为 0 时的名 义抗剪强度, $Z$ 可表示为

$Z=\frac{\tau_{\mathrm{u}}}{\tau_{\mathrm{u} 0}-1} \times 100 \%$ 


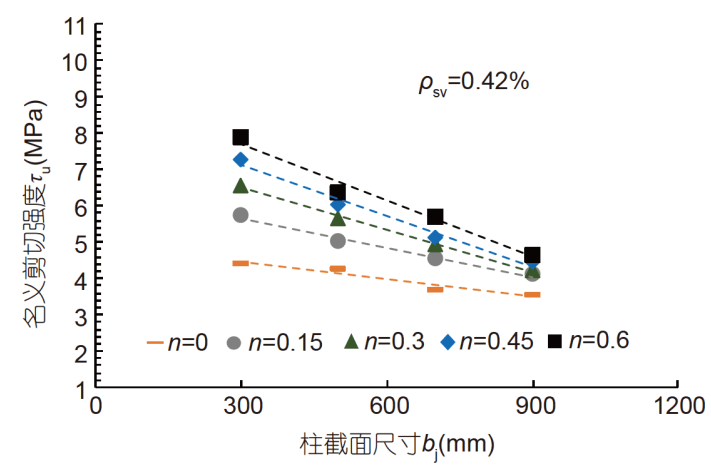

图 9 (网络版彩图)不同轴压比下名义抗剪强度与节点宽度 的关系

Figure 9 (Color online) The relationship between nominal shear strength and joint width under different axial compression ratios.

图10展示了不同尺寸的节点随轴压比的增大其名 义剪切强度提高百分比的变化趋势，横坐标为节点类 型，按照“节点宽度-轴压比”表示，如“300-0”即为节点 核心区宽度为 $300 \mathrm{~mm}$ 、轴压比为 0 的节点. 随着尺寸 增大，名义剪切强度提高百分比 $Z$ 降低，说明尽管轴压 比的增大可以提高节点的抗剪强度，但受到节点尺寸 效应的影响，其提高程度随尺寸增大而降低，也可以 理解为轴压比对节点抗剪强度的提高首先弥补了尺寸 效应造成的强度退化.

\section{3 配箍率影响}

节点核心区箍筋的存在不仅能够分担核心区混凝 土受到的剪力，同时也对发生横向膨胀的混凝土有约 束作用, 从而提高其抗压、抗剪强度.

图 11 为轴压比为 0.15 , 不同尺寸、不同配䈨率情

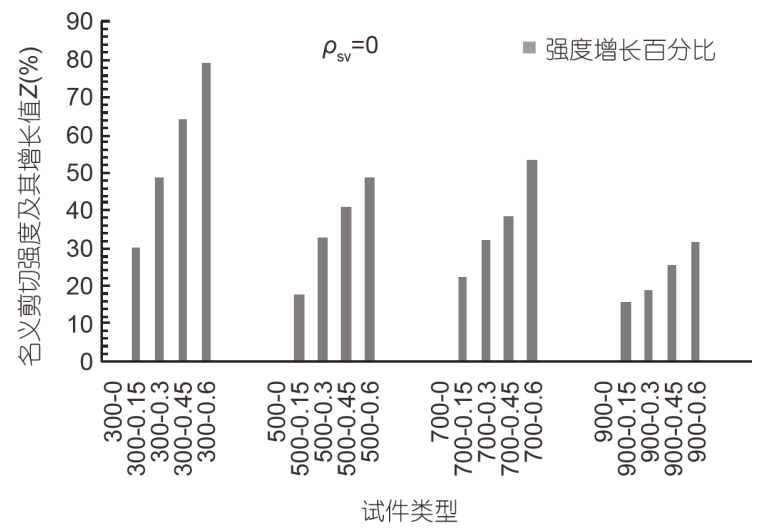

图 10 名义剪切强度提高程度

Figure 10 The increase of nominal shear strength.

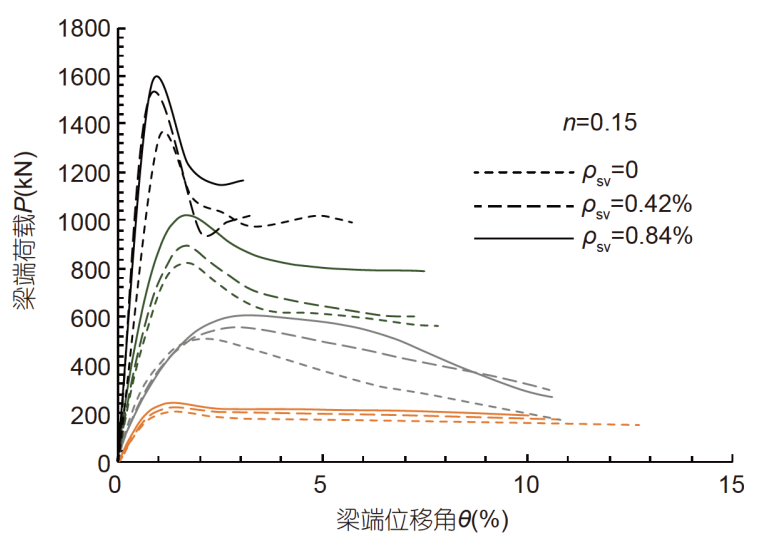

图 11 (网络版彩图)轴压比为 0.15 时梁端荷载 $P$-位移角 $\theta$ 曲 线对比

Figure 11 (Color online) Comparison of Load $P$-Displacement angle $\theta$ curves of beam end when the axial compression ratio is 0.15 .

况下的节点梁端荷载 $P$-位移角 $\theta$ 曲线图(尺寸沿纵坐标 依次增大). 从图中可以看出, 相同尺寸的节点, 其梁端 峰值荷载随配䈨率的增大略有提高, 配䈨率较大的试 件峰值荷载后的软化曲线相对平缓，即延性较好; 另 外, 随着节点尺寸的增大, 其峰值荷载提高, 位移角的 大小呈现递减趋势, 且变形能力亦随之下降.

图 12 为节点核心区宽度为 $500 \mathrm{~mm}$ 、轴压比 0.3 , 不 同配箱率情况下的节点损伤破坏图. 可以看出, 提高配 䈐率可以在一定程度上减轻破坏程度. 核心区䈐筋的 增多，一方面使节点的强度得以增强，另一方面也加 强了对混凝土的约束作用，核心区箍筋可以对节点抗 剪起到有利的作用. 当核心区配䈐率为 0 时，贯穿核心 区的梁筋及柱筋承受了较大拉力的作用，梁筋发生了 大量的屈服; 核心区配箍率为 $0.42 \%$ 及 $0.84 \%$ 时，箍筋 可以有效地分担剪力作用, 并改善梁筋的大的黏结-滑

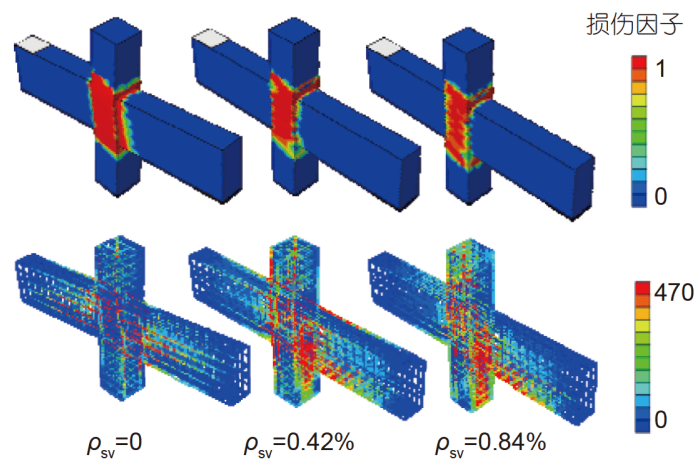

图 12 (网络版彩图)不同配箍率节点损伤破坏图

Figure 12 (Color online) Failure models of joints with different stirrup ratios. 
移行为.

图13为不同配箍率下，节点名义剪切强度随尺寸 变化的关系图(从图6中选取). 同一配䈨率下，节点名 义剪切强度随尺寸增大具有降低的趋势, 配䈨率的增 大使这种趋势得到缓解，说明配䈨率的增大将削弱尺 寸效应. 同时, 可发现在同一尺寸下, 配箙率的提高可 以增大节点的抗剪强度.

为直观地体现配䈐率对节点尺寸效应的削弱程 度, 本文定义尺寸效应程度值 $D_{\mathrm{s}}$ :

$D_{\mathrm{s}}=\frac{\tau_{\min }-\tau_{\max }}{\tau_{\min }} \times 100 \%$,

式中, $\tau_{\min }$ 为同种工况下节点核心区尺寸最小的节点的 名义剪切强度值，本文中为 $300 \mathrm{~mm} \times 300 \mathrm{~mm}$ 的节点； $\tau_{\max }$ 为同种工况下节点核心区尺寸最大的节点的名义 剪切强度值, 本文中为 $900 \mathrm{~mm} \times 900 \mathrm{~mm}$ 的节点.

尺寸效应程度值代表节点名义剪切强度值随尺寸 增大而降低的程度，程度值越大即代表尺寸效应越显 著. 图14为在不同轴压比、不同配筢率情况下得到的 节点核心区剪切强度的尺寸效应程度值对比图．可以 看出，在同一轴压比下，尺寸效应程度值随配䈨率增 大而降低，说明配䈐率的增大使得尺寸效应被削弱， 这是由于配箍率的提高可以改善节点的受力性能，包 括增大节点的抗剪强度和提高延性等. 尤其是对于大 尺寸构件而言，其混凝土强度受尺寸效应影响有所降 低，使得大尺寸构件在加载的中后期更加依赖于箍筋 的贡献(筢筋的参与程度、对混凝土的约束作用等), 因此配箍率的提高将削弱尺寸效应. 同时, 轴压比较大

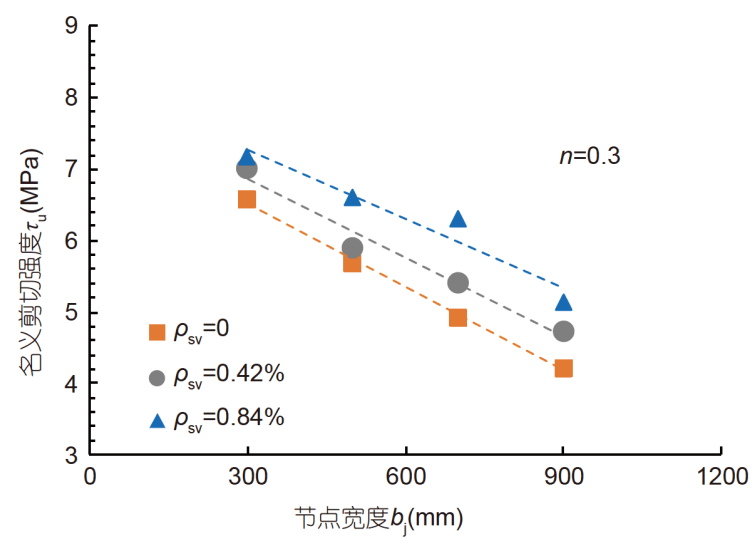

图 13 (网络版彩图)不同配筢率下名义剪切强度与节点宽 度的关系

Figure 13 (Color online) The relationship between nominal shear strength and joint width under different stirrup ratios.

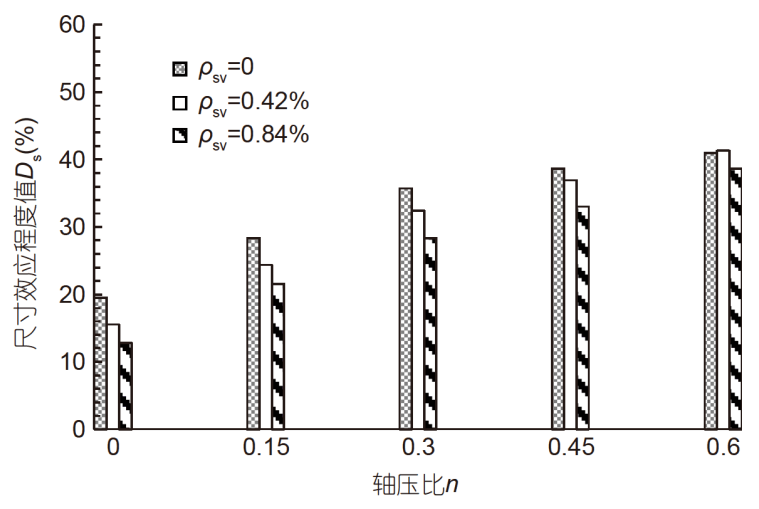

图 14 尺寸效应程度值对比

Figure 14 Contrast of size effect degree.

时, 配䈨率对尺寸效应程度值的削弱程度降低, 正如前 文所述，轴压比在加载初期能够抑制核心区裂缝的发 展速度, 但较大的轴压比会使核心区混凝土承受更多 的压力，使其提前发生压溃，破坏特征呈现更明显的 脆性, 配箍率的提高难以显著地抑制其脆性破坏, 故 而轴压比大的节点, 配箍率对尺寸效应的削弱程度较 低. 同时也可以看出, 轴压比增大时尺寸效应程度值 增大, 即轴压比增大时, 尺寸效应将增强.

\section{5 尺寸效应分析与讨论}

\section{1 剪切强度尺寸效应规律}

上述模拟结果的分析表明中节点剪切破坏存在明 显的尺寸效应行为, 且其受到轴压比和配䈨率的显著 影响. 目前, 研究者已建立了几种典型的尺寸效应律, 包括断裂力学尺寸效应律 ${ }^{[21]}$ 、统计尺寸效应律 ${ }^{[22]}$ 、 多重分形尺寸效应律 ${ }^{[23]}$ 、边界尺寸效应律 ${ }^{[24]}$. Bažant 基于断裂力学理论提出了适用于混凝土材料的尺寸效 应理论公式 ${ }^{[21]}$, 且大量的混凝土材料层次试验及模拟 证实了该尺寸效应律的合理性. 这里, 也对该尺寸效 应理论公式的合理性进行分析与讨论, 其给出的名义 剪切强度 $\tau_{\mathrm{u}}$ 的表达式:

$\tau_{\mathrm{u}}=\frac{B f_{\mathrm{t}}^{\prime}}{\sqrt{1+D / D_{0}}}$,

式中, $f_{\mathrm{t}}^{\prime}$ 为混凝土材料抗拉强度 (选取为䢃拉强度, 即为 $3.10 \mathrm{MPa}), B$ 和 $D_{0}$ 是依赖于结构的几何常数, $D$ 是试件 尺寸(这里选为节点高度).

将式(5)转化为线性方程: 
$Y=A X+C$,

式中, $Y=\left(f_{\mathrm{t}}^{\prime} / \tau_{\mathrm{u}}\right)^{2}, A=C / D_{0}, X=D, C=1 / B^{2}$.

本文使用以上尺寸效应律对节点模拟得出的名义 剪切强度值进行拟合，并与线弹性断裂力学理论结果 (LEFM，针对完全脆性材料)及弹性或塑性理论结果 (针对塑性材料, 不考虑尺寸效应)进行对比分析, 得出 如图15(a)所示的钢筋混凝土梁-柱节点名义剪切强度 随尺寸、轴压比变化的双对数曲线.

从图15(a)中可以看出，模拟数据点介于弹塑性理 论曲线和线弹性理论曲线之间, 即梁-柱中节点核心区 剪切破坏行为介于塑性破坏与脆性破坏之间. 另外, 可 以发现，模拟数据与Bažant尺寸效应律吻合良好(相关 系数 $\left.R^{2}=0.9\right)$, 说明Bažant尺寸效应律能够较好地描述 钢筋混凝土梁-柱中节点剪切破坏的尺寸效应规律.
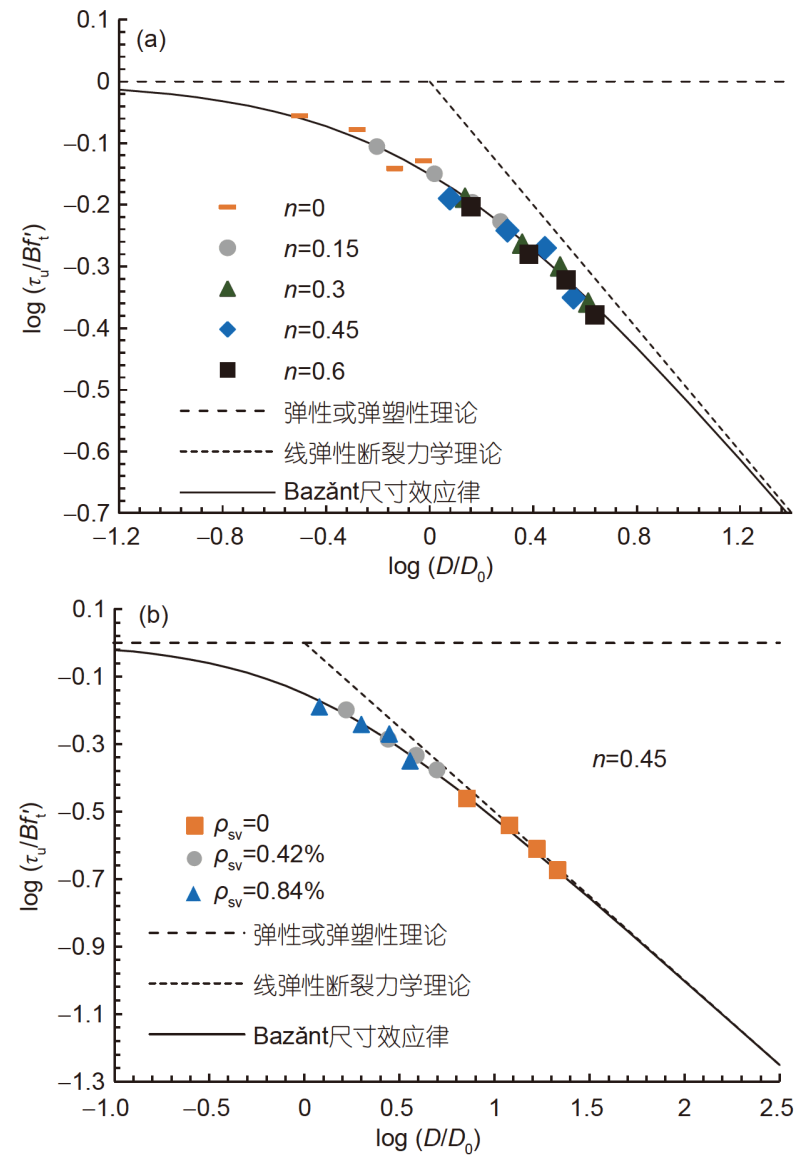

图 15 (网络版彩图)名义剪切强度双对数曲线. (a) 轴压比 影响; (b) 配筋率影响

Figure 15 (Color online) Double logarithmic curve of nominal shear strength. (a) Axial compression ratio; (b) stirrup ratio.
同时, 随着轴压比的增大, 数据点愈发靠近线弹性 断裂力学曲线, 即节点的破坏特征向着脆性破坏发展, 这也说明了尽管增大轴压比可以增强节点的抗剪承载 力, 但同时也会增强尺寸效应, 过大的轴压力会导致节 点更快地出现脆性破坏。

图15(b)为钢筋混凝土梁-柱中节点名义剪切强度 随尺寸、配筢率变化的双对数曲线. 可以看出, 配䇽率 小的数据点更接近线弹性断裂力学曲线, 即更接近脆 性破坏，尺寸效应越显著. 䈨筋的存在，限制了裂缝宽 度的扩展，使得骨料间咬合作用增强，强度增大，且削 弱名义剪切强度的尺寸效应行为.

\section{2 分析与讨论}

表 4 为不同工况条件下拟合得到的 $B$ 和 $D_{0}$ 值. 可以 发现，在不同配䇚率及不同轴压比下，拟合得到的 $B$ 与 $D_{0}$ 的值均明显不同. 实际上, 正如上文所述, 轴压比及 配䈨率对节点剪切破坏尺寸效应均有显著的影响, 故 而不同工况下 $B$ 和 $D_{0}$ 的大小与配䈨率和轴压比密切关 联, 即 $B$ 和 $D_{0}$ 应是其函数. 同时, 难以通过表 4 中的相关 数据, 总结和归纳出 $B$ 和 $D_{0}$ 与配箍率及轴压比之间的 定量关系.

这本质上说明，由于钢筋混凝土构件(如本文的

表 4 不同配筷率和轴压比下 $B$ 及 $D_{0}$ 的值

Table 4 Values of $B$ and $D_{0}$ under different stirrup ratios and axial compression ratios

\begin{tabular}{cccc}
\hline 配䈐率 $\rho_{\mathrm{sv}}$ & 轴压比 $n$ & $B$ & $D_{0}$ \\
\hline \multirow{3}{*}{0} & 0 & 5.16 & 625.00 \\
& 0.15 & 7.56 & 350.00 \\
& 0.3 & 13.25 & 95.00 \\
& 0.45 & 20.00 & 41.67 \\
$0.42 \%$ & 0.6 & 37.80 & 14.00 \\
\hline \multirow{3}{*}{$0.84 \%$} & 0 & 5.29 & 895.00 \\
& 0.15 & 7.45 & 450.00 \\
& 0.3 & 10.31 & 235.00 \\
& 0.45 & 11.79 & 180.00 \\
& 0.6 & 12.40 & 216.67 \\
\hline & 0.15 & 7.74 & 334.00 \\
& 0.3 & 9.21 & 295.00 \\
& 0.45 & 10.00 & 333.33 \\
& 0.6 & 11.55 & 250.00 \\
& & 12.70 & 206.67 \\
\hline
\end{tabular}


梁-柱节点)的破坏机制和失效模式远远复杂于混凝土 材料，适应于混凝土材料层次的Bažant尺寸效应律难 以全面地揭示构件层面的尺寸效应行为. 正如本文所 关注的梁-柱中节点破坏行为，Bažant尺寸效应律虽能 宏观的描述名义剪切强度随尺寸变化而变化的规律, 但其不能定量地描述其他因素，如轴压比和配䈨率等 对尺寸效应的影响. 因此, 在后续工作中需要对梁-柱 节点剪切破坏的尺寸效应理论作深入研究，发展能够 反映轴压比及配箍率等重要参数影响的尺寸效应理论 模型, 进而建立构件层次的尺寸效应律.

\section{6 结论}

本文结合已有钢筋混凝土梁-柱中节点抗震性能 试验研究, 借助细观数值模拟方法对节点核心区剪切 破坏机制与失效模式等进行了扩展分析，探究了更大 结构尺寸(节点最大尺寸为 $900 \mathrm{~mm} \times 900 \mathrm{~mm}$ )、更多轴
压比( $0 \sim 0.6)$ 及更多配箍率 $(0 \sim 0.84 \%)$ 情况下中节点剪 切破坏及尺寸效应行为. 得到以下结论.

（1）在单调荷载作用下，本文工况的梁-柱中节点 发生脆性剪切破坏，随尺寸的增大，名义剪切强度降 低, 存在显著的尺寸效应特征.

(2) 本文选取的轴压比 $(0 \sim 0.6)$ 范围内, 轴压比越 大, 节点抗剪承载力越大, 但破坏的脆性变强, 名义剪 切强度尺寸效应增强.

(3) 增大配䈨率可提高节点的抗剪承载力, 并对节 点抗剪强度的尺寸效应起到抑制或削弱作用.

（4）材料层次的Bažant尺寸效应律能宏观地反映 节点核心区剪切强度的尺寸效应规律，但其不能定量 地描述其他因素，如轴压比和配䈨率等对尺寸效应的 影响.

后续研究工作中，函待建立能够反映配筋率和轴 压比等重要因素影响的梁-柱节点名义剪切强度尺寸 效应律.

\section{参考文献}

1 Bird J F, Bommer J J. Earthquake losses due to ground failure. Eng Geol, 2004, 75: 147-179

2 van de Lindt J W, Goh G H. Effect of earthquake duration on structural reliability. Eng Struct, 2004, 26: 1585-1597

3 Research Group on Frame Joints. Shear strength of reinforced concrete beam-column joints under low reversed cyclic loading (in Chinese). J Build Struct, 1983, 4: 1-17 [框架节点专题研究组. 低周反复荷载作用下钢筋混凝土框架梁柱节点核心区抗剪强度的试验研究. 建筑结构 学报, 1983, 4: 1-17]

4 Fu J P, Chen A P, Chen X Y, et al. Experimental research on the seismic behavior of the interior joint with high shear-compression ratios in the frame (in Chinese). J Chongqing Jianzhu Univ, 2006, 28: 40-46 [傅剑平, 朱爱萍, 陈小英, 等. 框架高剪压比中间层中节点抗震性能试验研究. 重庆建筑大学学报, 2006, 28: 40-46]

5 Lü X L, Guo Z X, Wang Y X. Experimental study on seismic behavior of beam-column subassemblages in RC frame (in Chinese). J Build Struct, 2001, 22: 3-7 [吕西林, 郭子雄, 王亚勇. RC框架梁柱组合件抗震性能试验研究. 建筑结构学报, 2001, 22: 3-7]

6 Sadjadi R, Kianoush M R. Analytical modeling of the shear behavior of RC exterior beam-column joints. In: Annual Conference-Canadian Society for Civil Engineering. 2005. 1-10

7 Ghobarah A, El-Amoury T. Seismic rehabilitation of deficient exterior concrete frame joints. J Compos Constr, 2005, 9: 408-416

8 Abrams D P. Scale relations for reinforced concrete beam-column joints. ACI Struct J, 1987, 84: 502-512

9 Li Z B, Guo E W, Zhou X Y, et al. Seismic behaviors and size effects of large-scale interior beam-column joints of RC frames (in Chinese). China Civil Eng J, 2012, 45: 39-47 [李振宝, 郭二伟, 周锡元, 等. 大尺度RC梁柱节点抗震性能及尺寸效应试验研究. 土木工程学报, 2012, 45: 3947]

10 Kim J, LaFave J M. Joint shear behavior of reinfored concrete beam-column connections subjected to seismic lateral loading. Technical Report. University of Illinois, 2009

11 Barbhuiya S, Choudhury A M. A study on the size effect of RC beam-column connections under cyclic loading. Eng Struct, 2015, 95: 1-7

12 Choudhury A M, Deb S K, Dutta A. Study on size effect of fibre reinforced polymer retrofitted reinforced concrete beam-column connections under cyclic loading. Can J Civ Eng, 2013, 40: 353-360

13 Jin L, Miao L Y, Han J Y, et al. Size effect tests on shear failure of interior RC beam-to-column joints under monotonic and cyclic loadings. Eng 
Struct, 2018, 175: 591-604

14 Du X L, Jin L, Li D. A state-of-the-art review on the size effect of concretes and concrete structures ( II ): RC members (in Chinese). China Civil Eng J, 2017, 50: 24-44 [杜修力, 金汶, 李冬. 混凝土与混凝土结构尺寸效应述评 (II): 构件层次. 土木工程学报, 2017, 50: 24-44]

15 Kim S M, Abu Al-Rub R K. Meso-scale computational modeling of the plastic-damage response of cementitious composites. Cement Concrete Res, 2011, 41: 339-358

16 Lubliner J, Oliver J, Oller S, et al. A plastic-damage model for concrete. Int J Solids Struct, 1989, 25: 299-326

17 Lee J, Fenves G L. Plastic-damage model for cyclic loading of concrete structures. J Eng Mech, 1998, 124: 892-900

18 Genikomsou A S, Polak M A. Finite element analysis of punching shear of concrete slabs using damaged plasticity model in ABAQUS. Eng Struct, 2015, 98: 38-48

19 Du X L, Jin L, Ma G W. A meso-scale analysis method for the simulation of nonlinear damage and failure behavior of reinforced concrete members. Int J Damage Mech, 2013, 22: 878-904

20 Ministry of Housing and Urban-Rural Development of the People's Republic of China (MOHURD). Code for Design of Concrete Structures: GB 50010-2010 (in Chinese). Beijing: China Architecture \& Building Press, 2010 [中华人民共和国住房和城乡建设部. 混凝土结构设计规范: GB50010-2010. 北京: 中国建筑工业出版社, 2010]

21 Bažant Z P. Size effect in blunt fracture: Concrete, rock, metal. J Eng Mech, 1984, 110: 518-535

22 Carpinteri A, Ferro G. Size effects on tensile fracture properties: A unified explanation based on disorder and fractality of concrete microstructure. Mater Struct, 1994, 27: 563-571

23 Weibull W. A statistical distribution function of wide applicability. J Appl Mech, 1951, 18: 293-297

24 Carrère V, Conel J E. Recovery of atmospheric water vapor total column abundance from imaging spectrometer data around $940 \mathrm{~nm}-$ Sensitivity analysis and application to Airborne Visible/Infrared Imaging Spectrometer (AVIRIS) data. Remote Sens Environ, 1993, 44: 179-204

\title{
Numerical study on shear failure and the corresponding size effect of interior RC beam-column joints
}

\author{
JIN Liu, WEI Na, MIAO LiYue \& DU XiuLi \\ Key Laboratory of Urban Security and Disaster Engineering, Beijing University of Technology, Beijing 100124, China
}

The shear failure of the core zone of reinforced concrete (RC) beam-column joint has brittle characteristics, so the size effect of shear strength can be caused. Based on the existing experimental research results, the numerical test method is used to analyze the structural size, axial compression ratio, and stirrup ratio on the effect of failure mechanism and failure mode of the RC beam-column joint, and reveal their influence on the size effect of shear strength. The maximum cross-sectional dimension of the joint is $900 \mathrm{~mm} \times 900 \mathrm{~mm}$. The results show that: (1) under the monotonic loading, the beam-column nodes exhibit brittle shear failure in the core region, and the nominal shear strength has a significant size effect; (2) the increase in the axial compression ratio can enhance the shear capacity of the middle joint and also strengthen the size effect of shear strength; (3) an increase in the stirrup ratio can increase the shear capacity of the joint, but it can also weaken the size effect of the shear strength. Lastly, the classical size effect law proposed by Bažant can reflect the size effect of shear failure of the middle node, but it cannot describe the quantitative influence of the axial compression ratio and stirrup ratio.

interior RC beam-column joint, core zone, shear failure, size effect, axial load ratio, stirrup ratio, numerical simulation

doi: $10.1360 /$ N092018-00441 